\title{
Field-based screening identifies resistance to Sunn pest (Eurygaster integriceps) feeding at vegetative stage in elite wheat genotypes
}

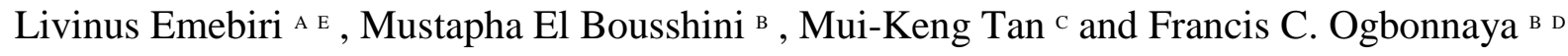 \\ ${ }^{\text {A }}$ Graham Centre for Agricultural Innovation (NSW Department of Primary Industries and Charles Sturt University), \\ Wagga Wagga, NSW 2650, Australia.
}

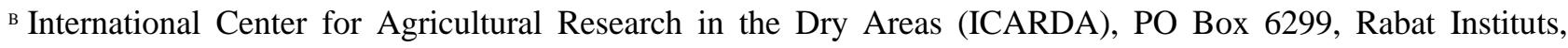
Morocco.

c NSW Department of Primary Industries, Woodbridge Road, Menangle, NSW 2568, Australia.

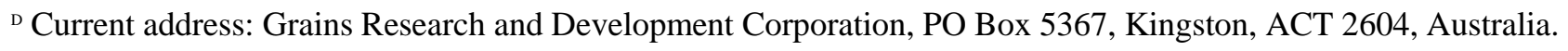

${ }^{\mathrm{E}}$ Corresponding author. Email: Livinus.Emebiri@dpi.nsw.gov.au

Crop and Pasture Science 68(2) 126-133 https://doi.org/10.1071/CP16355 Submitted: 26 September 2016 Accepted: 31 January 2017 Published: 25 February 2017

\section{Abstract}

Sunn pest (Eurygaster integriceps Puton) is currently widely distributed in West and Central Asia and Eastern Europe, but has not been found in Australia, Western Europe or North America. Climate warming is known to promote the expansion of its range of distribution, and it is expected that the insect could spread into new territories. Varieties of wheat (Triticum aestivum) carrying resistance remain an important component of managing the biosecurity risk of any potential incursion. Previous studies have identified sources of Sunn pest resistance in wheat, but there is little information on the genes that confer the resistance. This research used field-based, artificial infestation cages to evaluate 204 elite wheat varieties for Sunn pest resistance, at Terbol, Lebanon. A significant $(P<0.001)$ difference in resistance was observed among the wheat germplasm, with 19 varieties rated as resistant to moderately resistant and 17 as highly susceptible. Three of the elite varieties showed very little damage, a status similar to that of the resistant check, ICBW-209273. In parallel, the research carried out a genome-wide scan with single-nucleotide polymorphism (SNP) markers to identify chromosome regions and putative genes associated with resistance. Association mapping identified SNP markers with significant associations on chromosomes 2D, 4B and 5B. When these markers were projected onto the wheat population sequencing-based (POPSEQ) reference map, they tended to map close to the location of wheat height-reducing genes. The phenotypic variation explained by the identified markers ranged from $7 \%$ to $11 \%$, and collectively, they explained $23.9 \%$ of the variation or $45 \%$ of the generalised heritability. Marker-trait association was confirmed in two independent, doubled-haploid wheat populations, derived from crosses involving wheat landraces from Afghanistan, where Sunn pest is recognised as an endemic problem. In the two wheat populations, the analyses validated the strong association between wsnp_BF483640B_Ta_2_2 and resistance to Sunn pest damage at the vegetative stage. This study demonstrates existence of genetic resistance to 
Sunn pest feeding at the vegetative stage in elite wheat germplasm. The study also identified and validated SNP markers that could be useful tools for transfer of resistance into new wheat cultivars.

Additional keywords: genome-wide association study (GWAS), genetic resistance, pre-emptive breeding, reduced-height genes (Rht). 